\title{
Combination Weighting Method Based on Maximizing Deviations and Normalized Constraint Condition
}

\author{
Fulian Yin, Lu Lu, Jianping Chai and Yanbing Yang \\ Department of science and technology, Communication University of China \\ yinfulian@cuc.edu.cn,b-lucy-ll@hotmail.com,jp_chai@sina.com, \\ 862165191@qq.com
}

\begin{abstract}
When the weight of each attribute is determined in the multiple attribute decision making problems, calculated by the method of subjective values or objective values solely will cause the problem that weight coefficient is not reasonable. So the paper puts forward the weightingmethod which is based on maximizing deviations and normalized constraint condition. The method integrates the subjective and objective weighting information. On the one hand, the deviation between each weight vector which is determined by the various weighting method makes the maximum of its total deviation. On the other hand, the various evaluated object integrated value makes the maximum of its total evaluated value. Thus we establish a double objective optimization model. What's more, we deduce the weight calculation formula by solving the model. Finally we have an experimental analysis. It proves that the combination weighting methodcan reflect the relative importance of each indicators and the information that index itself contains. In other words, it can reflect the subjective and objective decisions which make the weighting results more reasonable.
\end{abstract}

Keywords: multiple attribute decision making; maximizing deviations; normalized constraint condition; combination weighting; weight

\section{Introduction}

Multiple attribute decision making is a kind of multi-objective decision making. It aims at limited scheme with multiple attributes, according to a decision criterion for scheme selection and sorting. It has extensive theoretical and practical application background in many fields such as engineer design, economy, management and military.[1] Multiple attribute decision making has some solution methods. These methods have a close relationship with the attribute weight. Because the rationality of weight affect the accuracy of multiple attribute decision making sorting, the weight plays an important part in the multiple attribute decision making.

Based on the differences between the source of raw date and the calculation process when we calculate the weight coefficient, the evaluation index weight determination method can be roughly divided into two types: subjective weighting method and objective weighting method. Subjective weighting method takes the method of qualitative. Experts make subjective judgment to get the weight according to the experience. Then they make a comprehensive evaluation to index, such as analytic hierarchy process [2], expert investigation method [3], the binomial coefficient method [4], sequential analysis method [5]. The analytic hierarchy process is the most common method used in the practical application. It makes complicated problems stratified and makes qualitative problems quantitative. Subjective weighting method can reflect the experience judgment of policy makers. The relative important degree of attributes doesn't violate people's common sense generally. While its randomness is bigger, the decision-making accuracy and reliability is a bit poor. According to the relationship of each index or the relationship 
between the index and evaluate results in the historical date, objective weighting method takes a comprehensive evaluate. It includes the entropy value method [6], principal component analysis [7], and the mean square deviation method [8]. The entropy value method is used frequently. The kind of weighting method uses data which is decision matrix. It determines the attribute weights reflect the degree of discrete attribute values. There is objective standard in the objective values. It can use certain mathematical model and get the coefficient weight of attribute by calculating. Ignorance of the subjective knowledge and experience of decision makers are its disadvantages. Sometimes the weight coefficient will be unreasonable. In view of the advantages and disadvantages of the subjective or objective weighting methods, there are a lot of discussions about the combination weighting method. Literature [9] proposed an assignment method based on a linear combination of entropy. Literature [10] puts forward the comprehensive weighting which combined AHP with expert investigation method and error back propagation neural network (BP work). Literature [11] according to optimization theory puts forward a kind of subjective preference and objective information linear comprehensive weighting method. Literature [12] constructs the comprehensive weighting method based on the sum of squared residuals. It uses the multi-attribute decision-making schemes value as the basic idea. Literature [13] combines subjective weights preference information which is given by decision makers with objective decision matrix information by a mathematical programming model. It makes sure the weights reflect the subjective and objective degree at the same time. Literature [14] deduces a comprehensive weighting method includes subjective preference and objective information which makes the analysis results of comprehensive weight grade method more reasonable and reliable. Literature [15] proposes the comprehensive integration weighting method based on the sum of square residuals andthe comprehensive integration weighting methodbased on normalized constraint condition respectively. Most combination weighting is based on optimization theory, and establishes the single objective optimization model and solves it.

When we need ensure attribute index weights in the multiple attribute decision making problem, calculating by subjective weighting method or objective weighting method singly will cause the problem that the weight coefficient is unreasonable. So the paper puts forward a comprehensive combination of subjective and objective weight information weighting approach. The method not only considers the decision makers' experience and judgment,but also reflects the raw date information and can reflect the subjective and objective decision at the same time. It eliminates the irrationality of using subjective or objective weighting alone in a certain degree.

\section{Combination Weighting Theory}

As various subjective and objective weighting method have advantages and disadvantages, scholars have put forward to combine various weight methods. There are two forms of the combination of the weighting method, multiplicative synthesis and linear weighted combination respectively.

Multiplicative synthesis method is making each index weight from weighting methods multiplication. Then we should normalize to get the combination weight. This method is suitable for more index and more uniform weight distribution.Linear weighted combination methodis to weight each weight form each weighting methods to get combination weight. When decision makers have preference in different weighting methods, weight can be determined by decision makers' preference. When decision makers have no preference in different weighting methods, there it needs to use other methods to determine the relative importance of different weighting method. Therefore, there is a weight allocation problem in the linear weighted combination.

Based on the linear weighted combination, the paper puts forward a combination method, which takes the optimization model to determine the weight allocation problem. 
Selection of weighting method, program and evaluation index

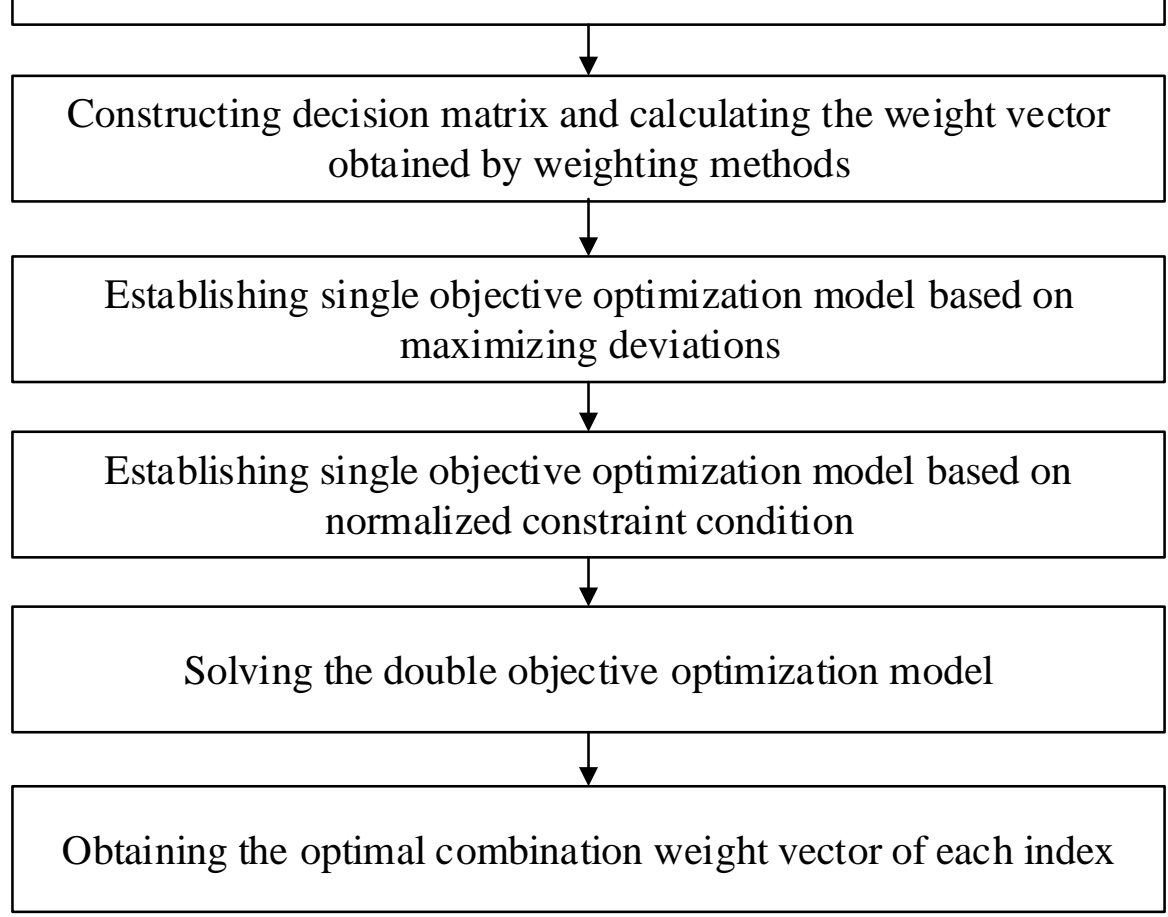

Figure 1. Flow Chart of Combination Weighting Method

\section{Combination Weighting Method Based on Maximizing Deviations and Normalized Constraint Condition}

In a multiple attribute decision making problem, the solution set is expressed as $S=\left\{S_{1}, S_{2}, \cdots, S_{m}\right\}$, the attributes (or index) set is expressed as $P=\left\{P_{1}, P_{2}, \cdots, P_{n}\right\}$, the attribute of the $i$ scheme $S_{i}$ accord to the $j$ index $P_{j}$ is expressed as $a_{i j}, i=1,2, \cdots, m, j=1,2, \cdots, n, \mathbf{A}=\left(a_{i j}\right)_{m \times n}$ is called decision matrix.

For a variety of different indicators, they can be generally divided into efficiency, cost, fixed type, and range. In order to eliminate the influence of different dimension to final decision matrix, we need to standardize decision matrix. The matrix $\mathbf{B}=\left(b_{i j}\right)_{m \times n}$ which has standardized is called standardized decision matrix. $b_{i j}$ means the standardized attribute value of the $i$ program $S_{i}$ accord to the $j$ index $P_{j}$. The $i$ line of matrixC means the standardized value of the $i$ program accord to $\mathrm{n}$ attribute values. Obviously the bigger $b_{i j}$, the better.

After weighting decision problem by using subjective weighting method and objective weighting method respectively, we assume that there is $l$ kinds of subjective and objective weighting methods to give $n$ indicators weight coefficients. The weight vector value of the $k$ weighting method is $\mathbf{W}_{\mathbf{k}}=\left(w_{1 k}, w_{2 k}, \cdots, w_{n k}\right)^{T}, k=1,2, \cdots, l, w_{j k} \geq 0$, $\sum_{j=1}^{n} w_{j k}=1, k=1,2, \cdots, l, j=1,2, \cdots, n$ 。 
For synthesize the characteristics of each weighting methods, we consider the following combination weighting:

$\mathbf{W}_{\mathbf{c}}=\theta_{1} \mathbf{W}_{\mathbf{1}}+\theta_{2} \mathbf{W}_{\mathbf{2}}+\cdots+\theta_{l} \mathbf{W}_{\mathbf{1}}$

$\mathbf{W}_{\mathbf{c}}=\left(w_{c 1}, w_{c 2}, \cdots, w_{c n}\right)^{T}$ is called combination weighting coefficient vector. $\theta_{1}, \theta_{2}, \cdots, \theta_{l}$ is called linear expressing coefficient of the combination weighting coefficient vector. Satisfying $\theta_{k} \geq 0, k=1,2, \cdots, l$, and normalized constraint condition

$$
\sum_{k=1}^{l} \theta_{k}^{2}=1
$$

$\mathbf{W}$ and $\boldsymbol{\Theta}$ can be expressed as partitioned matrix: $\mathbf{W}=\left(\mathbf{W}_{1}, \mathbf{W}_{2}, \cdots, \mathbf{W}_{\mathbf{1}}\right)$ and $\boldsymbol{\Theta}=\left(\theta_{1}, \theta_{2}, \cdots, \theta_{l}\right)^{T} . \mathbf{W}$ is called weight coefficient vector matrix which made in $l$ kinds of weighting methods. $\mathbf{W}$ is the $n \times l$ matrix in fact, $\boldsymbol{\Theta}$ is $l$ dimension column vector which made inlinear expressing coefficient of the combination weighting coefficient vector. Formula (1), (2) can be expressed as:

$$
\begin{aligned}
& \mathbf{W}_{\mathrm{c}}=\mathbf{W} \boldsymbol{\Theta} \\
& \boldsymbol{\Theta}^{\mathrm{T}} \boldsymbol{\Theta}=\mathbf{1}
\end{aligned}
$$

According to the simple linear weighted combination method, the comprehensive index of the $i$ program $S_{i}$ can be expressed as:

$$
Z_{i}\left(\mathbf{W}_{\mathbf{c}}\right)=\sum_{j=1}^{n} b_{i j} w_{c j}, \quad i=1,2, \cdots, m(5)
$$

Generally speaking, the biggerthe $Z_{i}\left(\mathbf{W}_{\mathbf{c}}\right)$ is, the better. That $Z_{i}\left(\mathbf{W}_{\mathbf{c}}\right)$ is bigger means the $i$ program $S_{i}$ is better.In the multiple attribute decision making problems, according to the comprehensive evaluation value of each decision scheme, we can take a comprehensive sequencing. Among them, the choice of the weight coefficient is very important.

In the comprehensive evaluation problem, we always want to make the comprehensive evaluation values of each decision-making scheme space out the difference generally. In other word, we hope to make the comprehensive evaluation values of each decision-making scheme be decentralized as much as possible. We also hope to maximize the comprehensive evaluation value of each evaluation objects.

\subsection{Make the Comprehensive Evaluation Values of each Decision-Making Scheme be Decentralized as much as Possible}

In order to maximize the total dispersion of all $\mathrm{n}$ indicators to all $\mathrm{m}$ decision-making plans,constructing the following objective function:

$$
J_{1}\left(\mathbf{W}_{\mathbf{c}}\right)=\sum_{j=1}^{n} \sum_{i=1}^{m} \sum_{i_{1}=1}^{m}\left|b_{i j}-b_{i_{1} j}\right| w_{c j}
$$

$n$ dimensional row vector $\mathbf{B}_{1}$ :

$$
\mathbf{B}_{\mathbf{1}}=\left[\sum_{i=1}^{m} \sum_{i_{1}=1}^{m}\left|b_{i 1}-b_{i_{1} 1}\right|, \sum_{i=1}^{m} \sum_{i_{1}=1}^{m}\left|b_{i 2}-b_{i_{1}}\right|, \cdots, \sum_{i=1}^{m} \sum_{i_{1}=1}^{m}\left|b_{i n}-b_{i_{1} n}\right|\right]
$$

The objective function can be expressed as:

$$
J_{1}\left(\mathbf{W}_{\mathbf{c}}\right)=\mathbf{B}_{1} \mathbf{W}_{\mathbf{c}}=\mathbf{B}_{\mathbf{1}} \mathbf{W} \Theta
$$

So we can establish the optimization model based on maximizing deviationI: 
$\max F_{1}(\boldsymbol{\Theta})=\mathbf{B}_{1} \mathbf{W} \Theta$

s.t. $\left\{\begin{array}{c}\boldsymbol{\Theta}^{T} \boldsymbol{\Theta}=1 \\ \boldsymbol{\Theta} \geq 0\end{array}\right.$

\subsection{Maximize the Comprehensive Evaluation Value of each Evaluation Objects}

The comprehensive evaluation values of the $i$ decision-making scheme $S_{i}$ can be expressed as:

$$
Z_{i}\left(\mathbf{W}_{\mathbf{c}}\right)=\sum_{j=1}^{n} b_{i j} w_{c j}, \quad i=1,2, \cdots, m
$$

In order to make the comprehensive evaluation value of each objects as large as possible, we can establish the optimization model:

$$
\begin{aligned}
& \max F_{2}^{*}(\boldsymbol{\Theta})=\left(Z_{1}\left(\mathbf{W}_{\mathbf{c}}\right), Z_{2}\left(\mathbf{W}_{\mathbf{c}}\right), \cdots, Z_{m}\left(\mathbf{W}_{\mathbf{c}}\right)\right) \\
& \text { s.t. }\left\{\begin{array}{l}
\boldsymbol{\Theta}^{T} \boldsymbol{\Theta}=1 \\
\boldsymbol{\Theta} \geq 0
\end{array}\right.
\end{aligned}
$$

Asthere is no preference relation between each decision scheme, we can get the answer by the weighted linear summation method that the multi-objective decision model is converted into equivalent single objective optimization model. The objective function, in other word, the comprehensive evaluation values of all the decision is

$$
J_{2}\left(\mathbf{W}_{\mathbf{c}}\right)=\sum_{i=1}^{m} Z_{i}\left(\mathbf{W}_{\mathbf{c}}\right)=\sum_{i=1}^{m} \sum_{j=1}^{n} b_{i j} w_{c j}
$$

$n$ dimensional row vector $\mathbf{B}_{2}$

$$
\mathbf{B}_{\mathbf{2}}=\left[\sum_{i=1}^{m} b_{i 1}, \sum_{i=1}^{m} b_{i 2}, \cdots, \sum_{i=1}^{m} b_{i n}\right]
$$

The objective function can be expressed

$$
J_{2}\left(\mathbf{W}_{\mathbf{c}}\right)=\mathbf{B}_{\mathbf{2}} \mathbf{W}_{\mathbf{c}}=\mathbf{B}_{\mathbf{2}} \mathbf{W} \boldsymbol{\Theta}
$$

So we can establish the optimization model based on the normalized constraint conditionII:

$$
\begin{aligned}
& \max F_{2}(\boldsymbol{\Theta})=\mathbf{B}_{2} \mathbf{W} \boldsymbol{\Theta} \\
& \text { s.t. }\left\{\begin{array}{c}
\boldsymbol{\Theta}^{T} \boldsymbol{\Theta}=1 \\
\boldsymbol{\Theta} \geq 0
\end{array}\right.
\end{aligned}
$$

\subsection{Solve the Double Objective Optimization Problem}

If we consider two constraints separately, we need to solve the optimization model Iand II. This paper will consider the two constraints mentioned above at the same time. On the one hand, it can make the comprehensive evaluation values of each decision-making scheme space out the difference. On the other hand, it can maximize it. This is a double objective plan problem. Using the linear weighted sum method solves the double objective optimization problem. The linear weighted sum method gets the weight according to the importance of each objective function, then gets the single objective function by summing the multiplication of the objective function and weight. The optimization problem is 


$$
\begin{aligned}
& \max F(\boldsymbol{\Theta})=\alpha \mathbf{B}_{\mathbf{1}} \mathbf{W} \boldsymbol{\Theta}+\beta \mathbf{B}_{\mathbf{2}} \mathbf{W} \boldsymbol{\Theta} \\
& \text { s.t. }\left\{\begin{array}{c}
\boldsymbol{\Theta}^{T} \boldsymbol{\Theta}=1 \\
\boldsymbol{\Theta} \geq 0
\end{array}\right.
\end{aligned}
$$

Among then, $\alpha+\beta=1$ 。

The corresponding weight shall be given with different values according to different situations. There is a calculating in the situation that the two optimization condition has the same importance. After such a weight assignment, we can establish double objective optimization model.

$$
\begin{aligned}
& \max F(\boldsymbol{\Theta})=0.5 \mathbf{B}_{\mathbf{1}} \mathbf{W} \boldsymbol{\Theta}+0.5 \mathbf{B}_{\mathbf{2}} \mathbf{W} \boldsymbol{\Theta} \\
& \text { s.t. }\left\{\begin{array}{c}
\boldsymbol{\Theta}^{T} \boldsymbol{\Theta}=1 \\
\boldsymbol{\Theta} \geq 0
\end{array}\right.
\end{aligned}
$$

$\mathbf{B}_{1}$ and $\mathbf{B}_{2}$ are dimensional row vectors, $\mathbf{W}$ is weight index vector matrix made in $l$ kind of weighting methods $l, \boldsymbol{\Theta}$ is 1 column vector made in the linear expressing coefficient of combination weight coefficient.

$$
\begin{aligned}
& \text { If } 0.5\left(\mathbf{B}_{\mathbf{1}}+\mathbf{B}_{\mathbf{2}}\right)=\mathbf{B}_{3} \text {, the optimization model is } \\
& \max F(\boldsymbol{\Theta})=\mathbf{B}_{3} \mathbf{W} \boldsymbol{\Theta} \\
& \text { s.t. }\left\{\begin{array}{c}
\boldsymbol{\Theta}^{T} \boldsymbol{\Theta}=1 \\
\boldsymbol{\Theta} \geq 0
\end{array}\right.
\end{aligned}
$$

Using the Lagrange multiplier method to solve the model. Construct the Lagrange function.

$$
L\left(\theta_{1}, \theta_{2}, \cdots, \theta_{l}\right)=\mathbf{B}_{3} \mathbf{W} \boldsymbol{\Theta}+\lambda\left(\boldsymbol{\Theta}^{\mathrm{T}} \boldsymbol{\Theta}-1\right)=\mathbf{B}_{3} \sum_{k=1}^{l} \mathbf{W}_{\mathbf{k}} \theta_{k}+\lambda\left(\sum_{k=1}^{l} \theta_{k}^{2}-1\right)
$$

$\lambda$ isLagrangian multiplier.Make $\partial L / \partial \theta_{k}=0, \mathbf{B}_{3} \mathbf{W}_{\mathbf{k}}+2 \lambda \theta_{k}=0, k=1,2, \cdots, l$. $\theta_{k}=-\mathbf{B}_{3} \mathbf{W}_{\mathbf{k}} / 2 \lambda, k=1,2, \cdots, l$

Take formula(20) into formula $\boldsymbol{\Theta}^{T} \boldsymbol{\Theta}=1$, conform to the formula $\boldsymbol{\Theta} \geq 0$ at the same time, then

$$
\lambda=-\sqrt{\sum_{k=1}^{l}\left(\mathbf{B}_{3} \mathbf{W}_{\mathbf{k}}\right)^{2}} / 2
$$

So the optimal solution of the double objective optimization model is

$$
\theta_{k}^{*}=\mathbf{B}_{3} \mathbf{W}_{\mathbf{k}} / \sqrt{\sum_{k=1}^{l}\left(\mathbf{B}_{3} \mathbf{W}_{\mathbf{k}}\right)^{2}}, k=1,2, \cdots, l
$$

Then take formula(22) into formula(1), we can get the optimization combination weighting vector of multiple attribute decision making based on the combination of maximizing deviations and normalized constraint condition.

$$
\mathbf{W}_{\mathbf{c}}^{*}=\boldsymbol{\theta}_{1}^{*} \mathbf{W}_{1}+\boldsymbol{\theta}_{2}^{*} \mathbf{W}_{2}+\cdots+\boldsymbol{\theta}_{1}^{*} \mathbf{W}_{1}
$$

Because of the normalized processing for weight vector, we need to take the normalized processing for $\mathbf{W}_{\mathbf{c}}^{*}=\left(w_{c 1}^{*}, w_{c 2}^{*}, \cdots, w_{c n}^{*}\right)^{T}$. We only need to take the normalized processing for $\theta_{k}^{*}, k=1,2, \cdots, l$. 


$$
\theta_{k}^{* *}=\theta_{k}^{*} / \sum_{k=1}^{l} \theta_{k}^{*}=\mathbf{B}_{3} \mathbf{W}_{\mathbf{k}} / \sum_{k=1}^{l}\left(\mathbf{B}_{3} \mathbf{W}_{\mathbf{k}}\right), k=1,2, \cdots, l
$$

Obviously, $\sum_{k=1}^{l} \theta_{k}^{* *}=1, \theta_{k}^{* *} \geq 0, k=1,2, \cdots, l$ 。

Take formula (24) into formula (1) .Get the optimization normalized combinationweighting vector of multiple attribute decision making based on the combination of maximizing deviations and normalized constraint condition.

$$
\mathbf{W}_{\mathbf{c}}^{* * *}=\boldsymbol{\theta}_{1}^{* * *} \mathbf{W}_{1}+\boldsymbol{\theta}_{2}^{* * *} \mathbf{W}_{2}+\cdots+\boldsymbol{\theta}_{1}^{* * *} \mathbf{W}_{1}
$$

\section{Experimental Analysis}

We choose Gehua viewing date in Beijing in March 2014 as the date source. Types in 14 shows as evaluation object set. Selecting six viewing index as a set of properties, $P_{1}, P_{2}, P_{3}, P_{4}, P_{5}, P_{6}$ They are audience rating(\%), arrival rating(\%), loyalty(\%), the market share $(\%)$, the average viewing time(minutes) and ratings section number(period). $P_{1}, P_{2}, P_{3}, P_{4}, P_{5}$ are quality-benefit type attributes, $P_{6}$ is cost attribute. Table 1 shows the original property value.

Table 1. Gehua viewing Date in Beijing in March 2014

\begin{tabular}{|c|c|c|c|c|c|c|}
\hline $\begin{array}{c}\text { program } \\
\text { type }\end{array}$ & $P_{1}$ & $P_{2}$ & $P_{3}$ & $P_{4}$ & $P_{5}$ & $P_{6}$ \\
\hline finance & 0.0013 & 0.2839 & 0.0048 & 0.0282 & 1076.4846 & 3.1349 \\
\hline tv series & 0.0021 & 0.5080 & 0.0041 & 0.2626 & 5604.5420 & 9.0750 \\
\hline moive & 0.0033 & 0.2849 & 0.0117 & 0.0406 & 1545.5377 & 2.9174 \\
\hline law & 0.0042 & 0.3020 & 0.0137 & 0.0321 & 1154.0796 & 2.9143 \\
\hline education & 0.0015 & 0.0669 & 0.0219 & 0.0030 & 481.2764 & 1.4299 \\
\hline other & 0.0015 & 0.5823 & 0.0025 & 0.0944 & 1757.7659 & 21.0363 \\
\hline teenagers & 0.0013 & 0.1868 & 0.0068 & 0.0189 & 1097.0800 & 3.4919 \\
\hline Life service & 0.0025 & 0.5578 & 0.0044 & 0.0874 & 1699.2490 & 13.8613 \\
\hline sports & 0.0032 & 0.3090 & 0.0105 & 0.0550 & 1929.8000 & 4.1186 \\
\hline drama & 0.0025 & 0.1647 & 0.0151 & 0.0113 & 743.1830 & 1.8426 \\
\hline news & 0.0036 & 0.4839 & 0.0075 & 0.1487 & 3331.5970 & 6.5669 \\
\hline music & 0.0018 & 0.2239 & 0.0079 & 0.0186 & 898.9922 & 2.1484 \\
\hline special & 0.0020 & 0.4606 & 0.0042 & 0.0942 & 2219.0070 & 5.4914 \\
\hline variety & 0.0029 & 0.4396 & 0.0067 & 0.1051 & 2592.4540 & 5.2894 \\
\hline
\end{tabular}

Set up original decision matrix by the data in table 1.Get normalized decision matrix by normalization formula according to the data in the literature [16].The standardized data is shown in table 2. 
Table 2. The Standardized Viewing Data

\begin{tabular}{|c|c|c|c|c|c|c|}
\hline $\begin{array}{c}\text { program } \\
\text { type }\end{array}$ & $P_{1}$ & $P_{2}$ & $P_{3}$ & $P_{4}$ & $P_{5}$ & $P_{6}$ \\
\hline finance & 0.0000 & 0.4210 & 0.1186 & 0.0971 & 0.1162 & 0.9130 \\
\hline tv series & 0.2759 & 0.8558 & 0.0825 & 1.0000 & 1.0000 & 0.6101 \\
\hline moive & 0.6897 & 0.4230 & 0.4742 & 0.1448 & 0.2077 & 0.9241 \\
\hline law & 1.0000 & 0.4562 & 0.5773 & 0.1121 & 0.1313 & 0.9243 \\
\hline education & 0.0690 & 0.0000 & 1.0000 & 0.0000 & 0.0000 & 1.0000 \\
\hline other & 0.0690 & 1.0000 & 0.0000 & 0.3521 & 0.2492 & 0.0000 \\
\hline teenagers & 0.0000 & 0.2326 & 0.2216 & 0.0612 & 0.1202 & 0.8948 \\
\hline Life service & 0.4138 & 0.9525 & 0.0979 & 0.3251 & 0.2377 & 0.3660 \\
\hline sports & 0.6552 & 0.4697 & 0.4124 & 0.2003 & 0.2827 & 0.8629 \\
\hline drama & 0.4138 & 0.1898 & 0.6495 & 0.0320 & 0.0511 & 0.9790 \\
\hline news & 0.7931 & 0.8091 & 0.2577 & 0.5612 & 0.5563 & 0.7380 \\
\hline mucis & 0.1724 & 0.3046 & 0.2784 & 0.0601 & 0.0815 & 0.9634 \\
\hline special & 0.2414 & 0.7639 & 0.0876 & 0.3513 & 0.3392 & 0.7928 \\
\hline variety & 0.5517 & 0.7231 & 0.2165 & 0.3933 & 0.4121 & 0.8032 \\
\hline Calculate & & & & \\
\hline
\end{tabular}

Calculate weight by coefficient of Variation, Entropy weight method, Mean-squared deviation decision, and Analytic hierarchy process respectively. When using AHP, we should choose three relative importance between policymakers index. The weighting results is shown in table 3 .

Table 3. The Evaluation Index Weight

\begin{tabular}{|c|c|c|c|c|c|c|}
\hline $\begin{array}{c}\text { inde } \\
\mathbf{x}\end{array}$ & $\mathbf{A H P ( 1 )}$ & $\mathbf{A H P ( 2 )}$ & $\mathbf{A H P ( 3 )}$ & $\begin{array}{c}\text { Coefficient } \\
\text { of } \\
\text { Variation } \\
\mathbf{W} 4\end{array}$ & $\begin{array}{c}\text { Entropy } \\
\text { weight } \\
\text { W5 }\end{array}$ & $\begin{array}{c}\text { Mean-squaredDeviatio } \\
\text { W W6 }\end{array}$ \\
\hline$P_{1}$ & 0.3871 & 0.3409 & 0.3409 & 0.1807 & 0.2082 & 0.1859 \\
\hline$P_{2}$ & 0.0627 & 0.0762 & 0.1226 & 0.1235 & 0.1019 & 0.1807 \\
\hline$P_{3}$ & 0.2467 & 0.2054 & 0.2054 & 0.1882 & 0.1886 & 0.1620 \\
\hline$P_{4}$ & 0.1588 & 0.2054 & 0.0762 & 0.2216 & 0.2419 & 0.1573 \\
\hline$P_{5}$ & 0.1014 & 0.1226 & 0.2054 & 0.2073 & 0.2047 & 0.1510 \\
\hline$P_{6}$ & 0.0433 & 0.0496 & 0.0496 & 0.0787 & 0.0546 & 0.1630 \\
\hline
\end{tabular}

According to the formula (7) and formula (13) to calculate the dimension row vector respectively.

$$
\begin{gathered}
\mathbf{B 1}=(67.9310,66.3163,55.9381,51.6448,48.5792,50.4893) \\
\mathbf{B 2}=(5.3448,7.6013,4.4742,3.6907,3.7853,10.7715)
\end{gathered}
$$

So,

$$
\mathbf{B 3}=(36.6379,36.9588,30.2062,27.6678,26.1823,30.6304)
$$

Take the weight vector from each weightingmethod into formula(24). We can get 
followings. $\theta_{1}^{* * *}=0.1703, \theta_{2}^{* * *}=0.1682, \theta_{3}^{* *}=0.1698, \theta_{4}^{* * *}=0.1625, \theta_{5}^{* * *}=0.1624$, $\theta_{6}^{* * *}=0.1668$. So, combination weight vector is

$$
\begin{aligned}
& \mathbf{W}_{\mathbf{c}}^{* *}=0.1703 \mathbf{W}_{1}+0.1682 \mathbf{W}_{\mathbf{2}}+0.1698 \mathbf{W}_{\mathbf{3}}+0.1625 \mathbf{W}_{\mathbf{4}}+0.1624 \mathbf{W}_{\mathbf{5}}+0.1668 \mathbf{W}_{\mathbf{6}} \\
& =(0.2753,0.1111,0.1997,0.1761,0.1649,0.0730)^{T}
\end{aligned}
$$

The evaluation results and rankings from each weighting method are shown in Table 4. In Table 4, S represents sore and $\mathrm{R}$ represents rank.

\begin{tabular}{|c|c|c|c|c|c|c|c|c|c|c|c|c|c|c|}
\hline \multirow{2}{*}{$\begin{array}{c}\text { typ } \\
\mathrm{e}\end{array}$} & \multicolumn{2}{|c|}{$\mathrm{AHP}(1)$} & \multicolumn{2}{|c|}{$\mathrm{AHP}(2)$} & \multicolumn{2}{|c|}{$\mathrm{AHP}(3)$} & \multicolumn{2}{|c|}{$\begin{array}{l}\text { Coefficien } \\
\quad t \text { of } \\
\text { Variation }\end{array}$} & \multicolumn{2}{|c|}{$\begin{array}{c}\text { Entropy } \\
\text { weight }\end{array}$} & \multicolumn{2}{|c|}{$\begin{array}{l}\text { Mean-squaredDeviatio } \\
\mathrm{n} \text { decision }\end{array}$} & \multicolumn{2}{|c|}{$\begin{array}{l}\text { combinatio } \\
\text { n weighting }\end{array}$} \\
\hline & $\mathrm{S}$ & $\mathrm{R}$ & $\mathrm{S}$ & $\mathrm{R}$ & $\mathrm{S}$ & $\mathrm{R}$ & $\mathrm{S}$ & $\mathrm{R}$ & $\mathrm{S}$ & $\mathrm{R}$ & $\mathrm{S}$ & $\mathrm{R}$ & $\mathrm{S}$ & $\mathrm{R}$ \\
\hline 1 & $\begin{array}{c}0.1 \\
2\end{array}$ & $\begin{array}{l}1 \\
4\end{array}$ & $\begin{array}{c}0.1 \\
4\end{array}$ & $\begin{array}{l}1 \\
3\end{array}$ & $\begin{array}{c}0.1 \\
5\end{array}$ & $\begin{array}{l}1 \\
3\end{array}$ & 0.19 & 13 & $\begin{array}{c}0.1 \\
6\end{array}$ & $\begin{array}{l}1 \\
3\end{array}$ & 0.28 & 13 & 0.17 & 13 \\
\hline 2 & $\begin{array}{c}0.4 \\
7\end{array}$ & 5 & $\begin{array}{c}0.5 \\
3\end{array}$ & 3 & $\begin{array}{c}0.5 \\
3\end{array}$ & 3 & 0.65 & 1 & $\begin{array}{c}0.6 \\
4\end{array}$ & 1 & 0.63 & 2 & 0.57 & 2 \\
\hline 3 & $\begin{array}{c}0.4 \\
9\end{array}$ & 3 & $\begin{array}{c}0.4 \\
7\end{array}$ & 4 & $\begin{array}{c}0.4 \\
8\end{array}$ & 4 & 0.41 & 6 & $\begin{array}{c}0.4 \\
0\end{array}$ & 6 & 0.49 & 6 & 0.46 & 6 \\
\hline 4 & $\begin{array}{c}0.6 \\
3\end{array}$ & 1 & $\begin{array}{c}0.5 \\
8\end{array}$ & 2 & $\begin{array}{c}0.6 \\
0\end{array}$ & 2 & 0.47 & 3 & $\begin{array}{c}0.4 \\
7\end{array}$ & 3 & 0.55 & 3 & 0.55 & 3 \\
\hline 5 & $\begin{array}{c}0.3 \\
2\end{array}$ & 9 & $\begin{array}{c}0.2 \\
8\end{array}$ & $\begin{array}{l}1 \\
0\end{array}$ & $\begin{array}{c}0.2 \\
8\end{array}$ & $\begin{array}{l}1 \\
0\end{array}$ & 0.28 & 10 & $\begin{array}{c}0.2 \\
6\end{array}$ & $\begin{array}{l}1 \\
0\end{array}$ & 0.34 & 10 & 0.29 & 10 \\
\hline 6 & $\begin{array}{c}0.1 \\
7\end{array}$ & $\begin{array}{l}1 \\
2\end{array}$ & $\begin{array}{c}0.2 \\
0\end{array}$ & $\begin{array}{l}1 \\
2\end{array}$ & $\begin{array}{c}0.2 \\
2\end{array}$ & $\begin{array}{l}1 \\
1\end{array}$ & 0.27 & 11 & $\begin{array}{c}0.2 \\
5\end{array}$ & $\begin{array}{l}1 \\
1\end{array}$ & 0.29 & 12 & 0.23 & 11 \\
\hline 7 & $\begin{array}{c}0.1 \\
3\end{array}$ & $\begin{array}{l}1 \\
3\end{array}$ & $\begin{array}{c}0.1 \\
4\end{array}$ & $\begin{array}{l}1 \\
4\end{array}$ & $\begin{array}{c}0.1 \\
5\end{array}$ & $\begin{array}{l}1 \\
4\end{array}$ & 0.18 & 14 & $\begin{array}{c}0.1 \\
5\end{array}$ & $\begin{array}{l}1 \\
4\end{array}$ & 0.25 & 14 & 0.17 & 14 \\
\hline 8 & $\begin{array}{c}0.3 \\
4\end{array}$ & 8 & $\begin{array}{c}0.3 \\
5\end{array}$ & 8 & $\begin{array}{c}0.3 \\
7\end{array}$ & 7 & 0.36 & 8 & $\begin{array}{c}0.3 \\
5\end{array}$ & 7 & 0.41 & 8 & 0.36 & 7 \\
\hline 9 & $\begin{array}{c}0.4 \\
8\end{array}$ & 4 & $\begin{array}{c}0.4 \\
6\end{array}$ & 5 & $\begin{array}{c}0.4 \\
8\end{array}$ & 5 & 0.42 & 5 & $\begin{array}{c}0.4 \\
2\end{array}$ & 5 & 0.49 & 5 & 0.46 & 5 \\
\hline 10 & $\begin{array}{c}0.3 \\
9\end{array}$ & 7 & $\begin{array}{c}0.3 \\
5\end{array}$ & 7 & $\begin{array}{c}0.3 \\
6\end{array}$ & 8 & 0.32 & 9 & $\begin{array}{c}0.3 \\
0\end{array}$ & 9 & 0.39 & 9 & 0.35 & 8 \\
\hline 11 & $\begin{array}{c}0.6 \\
0\end{array}$ & 2 & $\begin{array}{c}0.6 \\
1\end{array}$ & 1 & $\begin{array}{c}0.6 \\
2\end{array}$ & 1 & 0.59 & 2 & $\begin{array}{c}0.5 \\
9\end{array}$ & 2 & 0.63 & 1 & 0.60 & 1 \\
\hline 12 & $\begin{array}{c}0.2 \\
1\end{array}$ & $\begin{array}{l}1 \\
1\end{array}$ & $\begin{array}{c}0.2 \\
1\end{array}$ & $\begin{array}{l}1 \\
1\end{array}$ & $\begin{array}{c}0.2 \\
2\end{array}$ & $\begin{array}{l}1 \\
2\end{array}$ & 0.23 & 12 & $\begin{array}{c}0.2 \\
0\end{array}$ & $\begin{array}{l}1 \\
2\end{array}$ & 0.31 & 11 & 0.23 & 12 \\
\hline 13 & $\begin{array}{c}0.2 \\
9\end{array}$ & $\begin{array}{l}1 \\
0\end{array}$ & $\begin{array}{c}0.3 \\
1\end{array}$ & 9 & $\begin{array}{c}0.3 \\
3\end{array}$ & 9 & 0.37 & 7 & $\begin{array}{c}0.3 \\
4\end{array}$ & 8 & 0.43 & 7 & 0.34 & 9 \\
\hline 14 & $\begin{array}{c}0.4 \\
5\end{array}$ & 6 & $\begin{array}{c}0.4 \\
6\end{array}$ & 6 & $\begin{array}{c}0.4 \\
8\end{array}$ & 6 & 0.47 & 4 & $\begin{array}{c}0.4 \\
5\end{array}$ & 4 & 0.52 & 4 & 0.47 & 4 \\
\hline
\end{tabular}

Table 4. The Evaluation Results and Rankings

It can be concluded from the Table 4 that weighting resultswhich are obtained by different methods differ from each other. The combination weightingmethod based on the maximizing deviations and normalized constraint condition assembles the advantages of other methods, which renders the methods of combination weighting more completed and accurate. 
These methods obtain weights from different prospects. For example, AHP compares the initial weights according to the relative importance from each index, while the Entropy value methodentrusts the weight to each different target from the prospect of information volume that each target contains. In the Table 4, the combination weighting based on the maximizing deviations and normalized constraint conditioncan not only reflect subjective decision, but also simultaneously reflect the objective decision, and can effectively integrate other different methods.

Experimental results indicates that weights calculated by AHP, Coefficient variation, Entropy weight method and Mean squared error are not equal to each other, in which case it's tough to generate an final evaluation of the projects. The way of combination weighting proposed in this paper maximized the final evaluation value and the deviation between value of our method and that of other methods, which makes the final weight coefficient of evaluation stable and reasonable.

\section{Conclusion}

The confirmation of weight of the evaluation index is a vital link of multiple attribute decision making, whether the evaluation is reasonable plays a pivotal role on the scientific of evaluation result. The change of a certain weight will influence the overall judgment. Therefore, it must be scientific and objective to settle a weight. This paper primarily summarizes the confirmation of weights available and analyzes the meritsanddemerits of all the methods, and subsequently puts forward a new method towards the combination weightingthat conducts a combination of the objective and subjective weightinginformation. On the one hand, this method considers the deviation of weight vectors ascertained by different methods of weights, makes the overall deviation up to the maximum and scatters the degree of each integrated assessment of objects as much as possible; on the other hand, the method also considers the integrated assessment of objects, and generally the bigger the value is, the better the plan performs.Thus, in order to maximize the sum evaluation, an optimization model of double targets is built. The paper then solves the model and gives the formula of weights. The experimental analysis in the end indicates the rationality of the method.

\section{References}

[1] XU Nanrong, ZHONG Weijun. The Theory and Method of Scientific Decision-Making. Nanjing University Press, 1996.

[2] Sadeghi M. , Ameli A. An AHP Decision Making Model for Optimal Allocation of Energy Subsidy among Socio-economic Subsectorsin Iran. Energy Policy, 2012, 45(6): 24-32.

[3] ZHEN Changqing. Weighting Method of Multi Objective Decision Making. Systems Engineering-Theory \& Practice, 1987, 7(2): $16^{\sim} 24$

[4] CHENG Mingxi.Two Coefficient Weighted Sum Methodof Multi Objective Decision Making. Systems Engineering-Theory \& Practice, 1983, 3(4): 23 26

[5] LU Mingsheng. The Weight Coefficient of Multi Objective Decision Making. Systems Engineering-Theory \& Practice, 1986, 6(4): $77^{\sim} 78$

[6] YANG Yiyong,LIUYahui,WANGMan,JIRun,JIXuewu. Objective Evaluation Method of Steering Comfort Based on Movement Quality Evaluation of Driver Steering Maneuver. Chinese Journal of Mechanical Engineering, 2014, 05:1027-1037.

[7] Croux C, Filzmoser P, Fritz H. Robust sparse principal component analysis. Technometrics, 2013, 55: 202-214.

[8] ZHOU Zikang, NA Jisheng. Study on Comprehensive Multi Criteria Scheduling Problem. Modernization of Management, 1990, (5): 5 7

[9] WANG Zeyan, GU Hongfang,YI Xiaoxin. A Linear Combination Weighting Method Based on Entropy. Systems Engineering-Theory \& Practice, 2003, 23(3): 112 116.

[10] LIANG Jie, HOU Zhiwei.Comprehensive Weighting Method Based on AHP, Expert Survey Method and Neural Network Method. Systems Engineering-Theory \& Practice, 2001, 21(3): 59 63.

[11] [11]. WANG Zhongxing,MOU Qiong, LI Qiaoxing. Combination weighting Methodof Multi Objective Decision Making. Communication on Applied Mathematics and Computation, 2003, 17(2): 55 62 . 
[12] CHEN Huayou. Combination Determining Weights Method for Multiple Attribute Decision Making Based on Maximazing Deviations. Journal of Systems Engineering and Electronics,2004,02:194-197.

[13] FAN Zhiping, ZHANG Quan, MA Jian.An Integrated Approach to Determining Weights in Multiple Attribute Decision Making. Journal of Management Sciences in China, 1998, 1(3):50-53.

[14] TAO Juchun, WU Jianmin.New Study on Determining the Weight of Index in Synthetic Weight Mark Method. Systems Engineering-Theory \& Practice, 2001, 21(8):43-48.

[15] ZHENG Bin, TAGN Shande, SHI Ziguo. Scheme Optimization of River Regulation Based on Combination Weighting Method. Water Resources and Power,2010,04:113-115+118.

[16] WANG Yingming, Fu Guowei.Using Multi-objective Decision Making Method to Make Decision for Multi-attributes. Control and Decision, 1993, 8(1): $25 \sim 29$.

[17] Laura S. Evaluation of Conditional Weight Approximations for Two-Level Models, Communications in Statistics-Simulation andComputation, 2012, 41(2): 182-204.

[18] Ishizaka Alessio, Pearman Craig, Nemery Philippe. AHPSort: an AHP-based method for sorting problems. International Journal of Product ion Research.2012, 50(17): 4767-4784.

[19] RadivojevićGordana, Gajović Vladimir. Supply chain risk mode ling by AHP and Fuzzy AHP methods. Journal of Risk Research, 2014, 17 (3): 337- 352.

[20] [20]. Lu Z S, Zhang Y. An augmented Lagrangian approach for sparse principal component analysis. Math Program, 2012, 135: 149-193.

[21] QingxingDONG,Thomas L.SAATY. AN ANALYTIC HIERARCHY PROCESS MODEL OF GROUP CONSENSUS. Journal of Systems Science and Systems Engineering, 2014, 03:363-375.

[22] Ozgur M ARAZ. Integrating Complex System Dynamics of Pandemic Influenzawith a Multi-Criteria Decision Making Modelfor Evaluating Public Health Strategies. Journal of Systems Science and Systems Engineering, 2013, 03:319-339.

[23] Research on group expandable optimization decision-making model for construction program choice. Engineering Sciences, 2012, 02:84-89.

[24] XIAO Wenxian, WANG Junge, MA Xiaoqin. Self-adaptive Differential Evolution Algorithm and Its Application to Complex Optimazaton Problem, Journal of Harbin University of Science and Technology, 2015,01:71-74.

[25] LI Dandan, TIAN Chunwei, LI Baiyang, SUN Guanglu, KANG Jian, Network Traffic Classification Based on Subspace Clustering-based Method, Journal of Harbin University of Science and Technology, 2015, 02: 63-68.

[26] A.AnandaShankar,Anandakumar. K.R. Opinion and Trust Based Collaborative Decision Making Model for Healthcare [A]. IACSIT.Proceedings of 2012 2nd International Conference on Industrial Technology and Management (ICITM 2012).IACSIT: 2012:5. 
International Journal of Security and Its Applications

Vol. 10, No. 2 (2016) 